\title{
IN SITU DEGRADATION OF 11/0 POLYESTER (MERSILENE) SUTURE MATERIAL FOLLOWING CATARACT SURGERY
}

\author{
A. J. KING, J. DEANE and J. SANDFORD-SMITH \\ Leicester
}

\begin{abstract}
SUMMARY
Two hundred and ten patients ( 231 eyes) had their cataract sections closed with $11 / 0$ polyester suture material over a 4 year period. Their case sheets were assessed to determine the number of eyes from which sutures had been removed prior to discharge, and for what reasons these sutures were removed. Ninety-seven patients (107 eyes) with sutures left in situ were further assessed to determine whether they were suffering from any suturerelated symptoms and examined to determine the degree of clinical biodegradation experienced by the sutures. Six of these sutures were removed for electron microscopic examination. Of the 231 eyes initially included, 61 sutures (26.4\%) had been removed prior to discharge. None of the 107 sutures examined showed evidence of clinical biodegradation; this was confirmed by electron microscopic analysis which showed only minimal erosion of sutures even after 48 months in situ.
\end{abstract}

Cataract operations are the most common intraocular procedure carried out in the United Kingdom. At present a variety of suture materials is available for closure of the section following cataract extraction, the most frequently used material being nylon. It is known that nylon undergoes extensive biodegradation in situ which may lead to a variety of ocular complications ranging from simple irritation and recurrent conjunctivitis to giant papillary conjunctivitis. ${ }^{1-4}$ As a consequence of these complications one of the authors (J.S.S.) changed to using $11 / 0$ polyester (Mersilene) sutures routinely, as these sutures are not considered to degrade with time. As the tensile strength of polyester suture material is greater than that of nylon the $11 / 0$ gauge was found to be adequate. In this paper we review the complications associated with use of 11/0 polyester sutures left in situ following cataract surgery, especially those attributed to biodegradation.

\section{METHODS}

Patients admitted under one consultant (J.S.S.) who had

Correspondence to: Mr A. J. King, Lecturer, Department of Ophthalmology, Leicester Royal Infirmary, Leicester LE2 7LX, UK. cataract extraction with intraocular lens implantation carried out between June 1990 and October 1991 were identified from the theatre register. Procedures carried out by all grades of doctor from Consultant to $\mathrm{SHO}$ were included for review. All patients had a peripheral corneal section closed with $11 / 0$ polyester in a continuous bootlace pattern with burying of the knot. Post-operatively only those patients with 2.5 dioptres or more of with-therule astigmatism had their sutures removed. Pre-operative and post-operative astigmatism were assessed by refraction and were considered with-the-rule if the axis of the positive cylinder was between $45^{\circ}$ and $135^{\circ}$. Review of the case sheets identified those patients in whom $11 / 0$ polyester material had been used. Deceased patients were excluded and the remaining case sheets further analysed to determine whether the suture material had been removed and, if so, for what reason and at what interval following the operation. The patients in whom the sutures had not been removed were identified and their general practitioners contacted as to their status. Patients who were deceased, who had moved out of the area or who were bedbound were excluded. The remaining patients with sutures left in place were requested to attend for review to determine whether they were suffering from any problems which could have been attributable to their suture material. These patients were examined to determine what changes the in situ sutures had undergone. All patients were interviewed and examined by the same doctor (A.K.).

A second group of patients with $11 / 0$ sutures in situ were recruited randomly from the clinic. These patients had their surgery after the original recruitment period and provided information on suture material left in situ for a shorter period of time (minimum follow-up 12 months). The ages and gender ratios of each group were statistically analysed to ensure no bias was introduced by the selection methods used to choose the patient groups.

At the review visit several patients had sutures removed. These sutures were examined with a scanning

Eye (1994) 8, 676-679 C 1994 Royal College of Ophthalmologists 
Table I. Surgically induced astigmatism

\begin{tabular}{lcc}
\hline Type of astigmatism & Number & $\%$ \\
\hline High with-the-rule ( $>3.0$ dioptres) astigmatism & 20 & 13.3 \\
Low with-the-rule (0.25-2.75 dioptres) astigmatism & 46 & 30.4 \\
No astigmatism & 17 & 11.2 \\
Low against-the-rule (0.25-2.75 dioptres) & 54 & 35.7 \\
$\quad$ astigmatism & & \\
High against-the-rule ( $>3.0$ dioptres) astigmatism & 14 & 9.3 \\
\hline
\end{tabular}

electron microscope to detect any ultrastructural changes experienced by the suture material.

\section{RESULTS}

From the theatre register, two hundred and ten patients representing 231 eyes were identified as having their cataract sections closed with $11 / 0$ polyester suture material. Of the 231 eyes reviewed, corrected visual acuities were $6 / 12$ or better in $195(84 \%)$ of the eyes, only 36 eyes failing to achieve this standard (age-related macular degeneration 20, myopic degeneration 4, diabetic maculopathy 3 , retinal detachment 2 , amblyopia 2 , glaucoma 1 , optic atrophy 1 , retinopathy of prematurity 1 , macular hole 1 , corneal scar 1). Forty-three had post-operative unaided visual acuities of $6 / 12$ or better.

Where available, pre-operative and 2 months post-operative refraction were assessed to determine any operatively induced astigmatic change. One hundred and fifty-one eyes had sufficient pre-operative and post-operative refractive data to allow this analysis. Sixty-six eyes had operatively induced with-the-rule astigmatism, ranging from 0.25 dioptres to 8.0 dioptres of astigmatism with a mean of 2.14 dioptres; 20 eyes had 3.0 dioptres or more of with-the-rule astigmatism. Sixty-eight eyes had operatively induced against-the-rule astigmatism which ranged from 0.25 dioptres to 4.0 dioptres of astigmatism with a mean of 1.9 dioptres; 14 eyes had 3.0 dioptres or more of against-the-rule astigmatism. Seventeen patients had no difference between their pre-operative and postoperative astigmatism. The mean alteration in astigmatism for the group was 1.79 dioptres prior to post-operative suture removal. Over three quarters $(77.4 \%)$ of patients had their astigmatism in the acceptable range of less than 3 dioptres with or against the rule before any suture removal (Table I).

Of the 231 eyes, 61 had their sutures removed prior to their final clinic visit and discharge. Twelve of these sutures were removed 'routinely' prior to discharge by doctors not aware of the policy of not removing sutures (Table II). A further 24 patients were excluded from

Table II. Reasons for suture removal prior to discharge

\begin{tabular}{lccll}
\hline Reason & Number & $\begin{array}{c}\text { Time } \\
\text { (months) }\end{array}$ & Other & \\
\hline Loose suture & 18 & $2-20$ & Proud suture & 4 \\
High cylinder & 20 & $2-9$ & Irritable eye & 2 \\
Routine & 12 & $3-18$ & Vascularisation & 2 \\
Other & 11 & $2-11$ & Sore eye & 2 \\
& 61 & & Contact lens fitting & 1 \\
\hline
\end{tabular}

review following consultation with their general practitioner (12 had died, 10 moved away and 2 were bedbound). One hundred and thirty-nine patients remained and of these 125 were requested to attend for examination; 65 of these did so, allowing examination of 74 eyes. Thirty-three patients recruited randomly from clinics were interviewed about suture-related problems and had their sutures examined. In all 107 eyes underwent examination from the two recruited groups. Statistical analysis showed no significant difference in age ( $p=0.63$; Mann-Whitney) or sex ( $p=0.24$; chi-squared) between the two groups.

The 107 examined eyes were divided into three groups according to the length of follow-up at the time of suture examination (Table III). Group I consisted of sutures between 12 and 24 months old, group II consisted of sutures between 25 and 36 months old, and group III consisted of sutures between 37 and 48 months old. In all, 11 patients had either symptoms potentially attributable to their sutures or sutures that required removal (Table IV).

Six patients had sutures removed for electron microscopic examination. The earliest changes are seen at 22 months, when slight cracking of the suture material is present. By 41 months the cracks have become more pronounced and some grooving is evident (Fig. 1).

\section{DISCUSSION}

In our series we assessed the outcome in 231 eyes which underwent routine cataract surgery having wound closure with $11 / 0$ polyester suture material. Our results show that only 43 of the eyes achieved post-operative visual acuity greater than $6 / 12$ unaided. This is accounted for mainly by the fact that in many cases when choosing an intraocular lens we aim to balance our final refractive correction with that of the other eye rather than aiming for emmetropia.

Only 17 of the 151 eyes assessed showed no difference between pre-operative and post-operative astigmatism. Of those that did alter, almost equal numbers showed changes to with-the-rule or against-the-rule astigmatism. This even spread of astigmatic change indicates the difficulty encountered when attempting to predict the correct suture tightness for wound closure.

Sixty-one of the eyes had their sutures removed in the routine follow-up period, representing a $26.4 \%$ removal rate. Considering that 32 of these cases had their sutures removed for high astigmatic cylinders or routinely as part of their pre-discharge protocol, this means that only $12.6 \%$ of cases had sutures removed for suture-related problems. This emphasises the low post-operative complication rate associated with the use of polyester suture material.

Table III. Data on sutures examined

\begin{tabular}{lcccc}
\hline & & \multicolumn{2}{c}{ Age of sutures (months) } & \\
\cline { 3 - 4 } & Number & Range & Mean & No. removed \\
\hline Group I & 22 & $12-24$ & 18.4 & 3 \\
Group II & 51 & $25-36$ & 31.2 & 8 \\
Group III & 34 & $37-48$ & 40.6 & 0 \\
\hline
\end{tabular}


Table IV. Reasons for removal of sutures at review

\begin{tabular}{llclll}
\hline Symptoms & Findings & Number & $\begin{array}{c}\text { Age of suture } \\
\text { (months) }\end{array}$ & Action & Change in symptoms \\
\hline Grittiness & Healthy suture. Blepharitis & 2 & 24,31 & Removal of suture & None \\
Grittiness & Loose suture with mucus trapping. Blepharitis & 1 & 25 & Removal of suture & None \\
Grittiness & Healthy suture. Blepharitis. Dry eyes & 1 & 27 & Removal of suture & None \\
None & Loose suture with mucus trapping & 5 & $14,15,28,30,33$ & Removal of suture & None \\
None & Loose suture & 1 & 29 & Removal of suture & None \\
None & Abraded suture & 1 & 33 & Removal of suture & None \\
\hline
\end{tabular}

Of the 107 eyes examined by us we found no evidence of clinical suture degradation in any case (Fig. 2) (longest follow-up time 48 months). At follow-up, however, we did elect to remove the sutures from 11 of the eyes. Only 4 of these patients were symptomatic; all were suffering from blepharitis and one was also suffering from dry eyes. Three of them had healthy-looking sutures and in one the suture was loose. Removal of the suture did not alter the symptoms in any of the patients. The remaining 7 patients were all asymptomatic; 6 had loose sutures usually associated with mucus trapping (Fig. 3) and one had an abraded suture probably due to handling at the time of wound closure. Even though only 65 of the 125 patients requested to attend did so, it is likely that patients who were sympto-

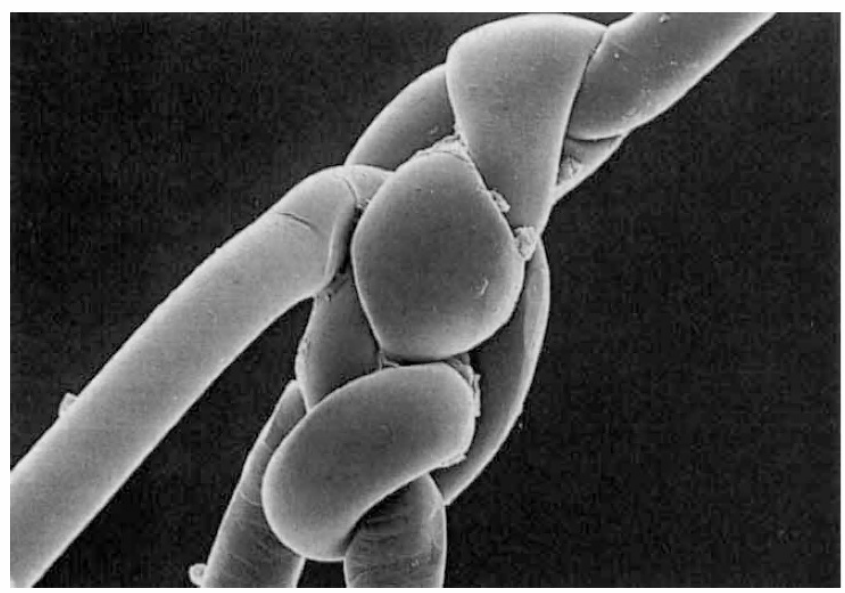

(a) matic would take the opportunity to undergo an ophthalmic assessment if offered. We therefore feel it is unlikely that we have missed any symptomatic patients in the group. These results compare very favourably with previous results for nylon sutures left in situ which showed $90 \%$ of nylon sutures to have undergone degradation after 3 years and $50 \%$ of patients to be symptomatic due to their degraded suture. ${ }^{5}$

It has been pointed out by several authors that nylon sutures can lead to ocular morbidity ranging from minor irritation to giant papillary conjunctivitis ${ }^{5-8}$ which thus necessitates removal of the suture. Removal of sutures routinely at 3-12 months post-operatively has therefore been advocated by several authors. ${ }^{5.6}$ This may involve an

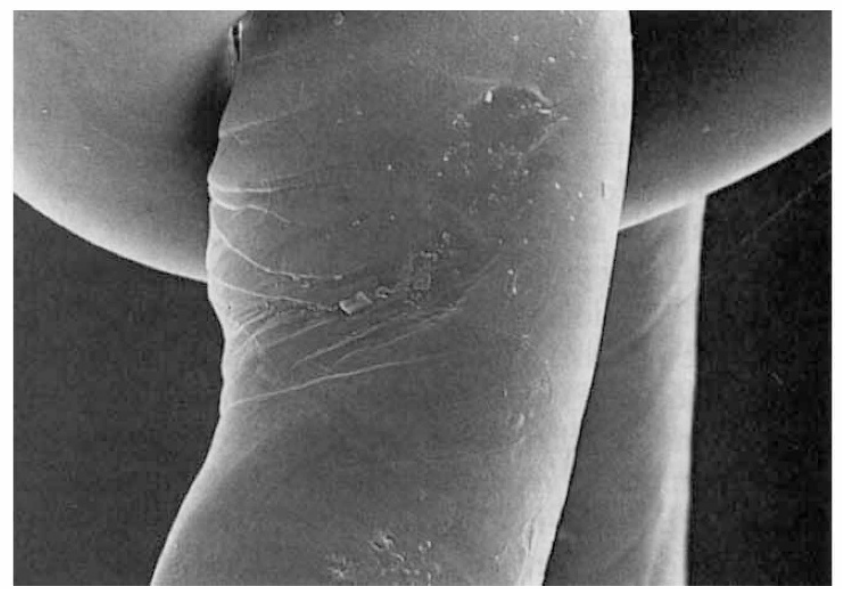

(b)

Fig. 1. Electron micrographs showing a suture removed at 48 months. (a) Cracks are seen on several of the suture limbs leaving the knot. (b) Magnification of one of the limbs shows evidence of grooving with thinning of the suture.

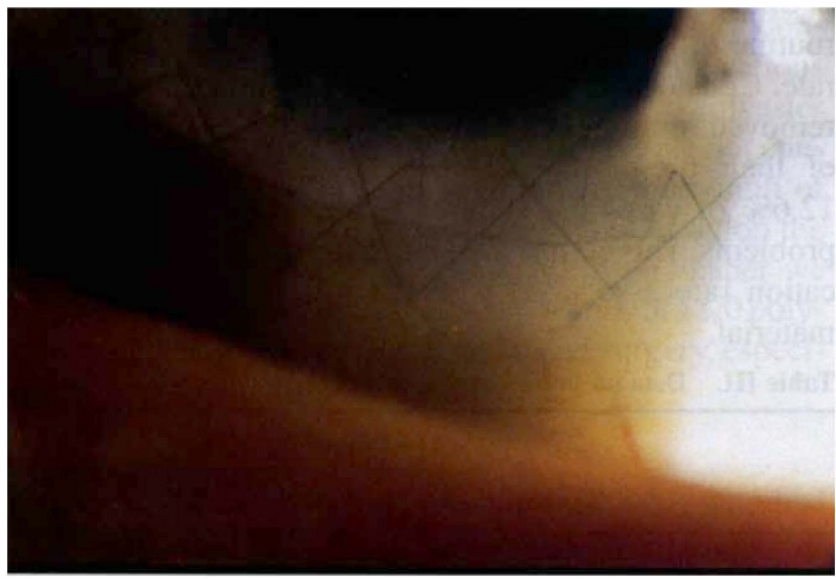

Fig. 2. Polyester suture material 48 months following surgery with no clinical evidence of degradation.

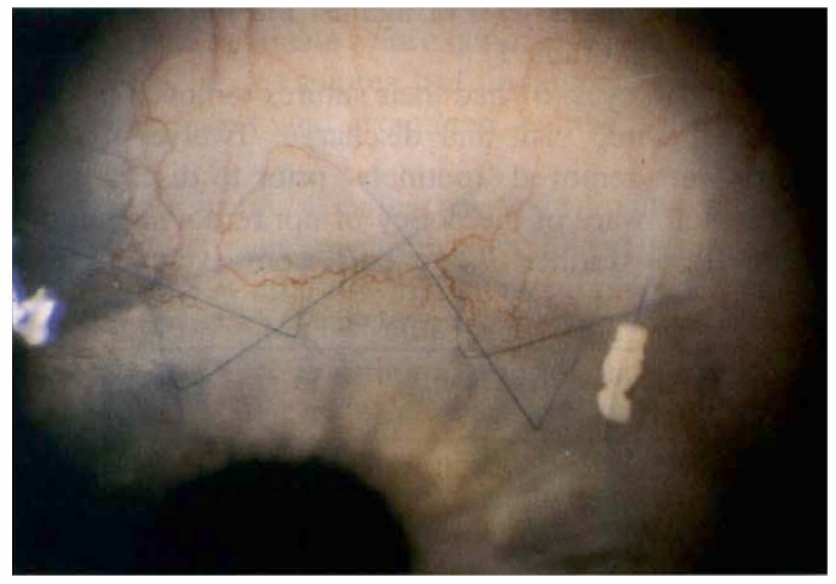

Fig. 3. Slightly loose suture with trapping of calcified plaque at 36 months. 
extra clinic visit which delays the patient's discharge from hospital and exposes them to a minor but sometimes difficult ocular procedure. There is also a risk of altering refraction following early suture removal. The alternative of leaving the nylon suture in place may expose the patient to significant discomfort and necessitate a visit to an eye casualty department. This causes considerable inconvenience and expense to the patient and often overburdens an already stretched department. Acheson et $_{\text {al. }}{ }^{6}$ have shown that up to $14.4 \%$ of eye casualty visits are due to suturerelated problems.

Electron microscopic examination has shown a minimal degree of biodegradation considering the period of time the suture had remained in situ; this supports the macroscopic findings. However, the presence of microscopic degradation does imply that polyester will also with time undergo biodegradation sufficient to cause suture disintegration. Our findings show this to be a very slow process and it is unlikely that these changes will be complete to trouble the average cataract patient during their lifetime.

This study has shown that in situ polyester suture material undergoes no clinically detectable degradation and minimal microscopic degradation over the duration of a follow-up period of up to 48 months. It is not associated with suture breakage and consequent ocular complications. In the few instances that the suture became loose, the majority of patients were completely asymptomatic. The use of $11 / 0$ polyester sutures therefore greatly reduces the risk of suture-related morbidity, thereby avoiding the need for routine suture removal.

\section{CONCLUSIONS}

Polyester suture material undergoes no clinically detectable and minimal microscopic biodegradation when left in situ following cataract surgery. This greatly reduces the risk of suture-related complications. By avoiding routine removal of sutures and minimising return visits to casualty both patient and doctor benefit.

We acknowledge the help of Jan Johnson and Hugh Harris, Photographers, Department of Ophthalmology, Leicester Royal Infirmary, and George McTurk, Department of Electronmicroscopy, University of Leicester.

Key words: Electron microscopy, In situ, Polyester, Suture complications, Suture degradation.

\section{REFERENCES}

1. Kronenthal RL. Intraocular degradation of non-absorbable sutures. Am J Intraocular Implant Soc 1977;3:222-4.

2. Cohan BE, Pearch AC, Schwartz S. Broken nylon fixation sutures. Am J Ophthalmol 1979;88:982-9.

3. Haysaka S, Ishiguro S, Shiono T, Okabe H, Mizuno K. A scanning electron microscopic study of nylon degradation by ocular tissue extracts. Am J Ophthalmol 1982;93:111-7.

4. Danjoux JP, Reck AC. Corneal sutures: is routine removal really necessary? Eye 1994;8:339-42.

5. Jackson H, Bosanquet R. Should nylon corneal sutures be routinely removed? Br J Ophthalmol 1991;75:663-4.

6. Acheson JF, Lyons CJ. Ocular morbidity due to monofilament nylon corneal sutures. Eye 1991;5:106-12.

7. Shahinian L, Brown SI. Postoperative complications with protruding monofilament nylon sutures. Am J Ophthalmol 1977;83:546-8.

8. Verinder S, Nirankari MD, Karesh JW, Richards RD. Complications of exposed monofilament sutures. Am J Ophthalmol $1983 ; 95: 515-9$. 